\title{
A STUDY OF ORTHOGONALITY OF BOUNDED LINEAR OPERATORS
}

\author{
TAMARA BOTTAZZI ${ }^{1}$, CRISTIAN CONDE ${ }^{2,3}$ AND DEBMALYA SAIN ${ }^{4}$
}

\begin{abstract}
We study Birkhoff-James orthogonality and isosceles orthogonality of bounded linear operators between Hilbert spaces and Banach spaces. We explore Birkhoff-James orthogonality of bounded linear operators in light of a new notion introduced by us and also discuss some of the possible applications in this regard. We also study isosceles orthogonality of bounded (positive) linear operators on a Hilbert space and some of the related properties, including that of operators having disjoint support. We further explore the relations between Birkhoff-James orthogonality and isosceles orthogonality in a general Banach space. Birkhoff-James orthogonality and isosceles orthogonality and norm attainment set and disjoint support [2010]Primary: 47A63, 51F20. Secondary: 47L05, 47A30.
\end{abstract}

\section{INTRODUCTION AND PRELIMINARIES}

The primary purpose of the present paper is to explore orthogonality of bounded linear operators between Hilbert spaces and Banach spaces. Unlike the Hilbert space case, there is no universal notion of orthogonality in a Banach space. However, it is possible to have several notions of orthogonality in such space, each of which generalizes some particular aspect of Hilbert space orthogonality. Indeed, one of the root causes of the vast differences between the geometries of Hilbert spaces and Banach spaces is the lack of a standard orthogonality notion in the later case. On the other hand, this makes the study of orthogonality of bounded linear operators an interesting and deeply rewarding area of research. Motivated by this, several authors have explored orthogonality of bounded linear operators in recent times [1], [4], [6], [7], [9], [10], [18, [19], [20], [21], [23], [24], 25], [27], [28] and [29], and have obtained many interesting results involving the geometry of operator spaces. In this paper, among other things, we extend, improve and generalize some of the earlier results on orthogonality of bounded linear operators. Without further ado, let us first establish our notations and terminologies to be used throughout the paper.

2010 Mathematics Subject Classification. Primary: 47A30, 47A63. Secondary: 47L05.

Key words and phrases. Birkhoff-James orthogonality; isosceles orthogonality; norm attainment set; disjoint support. 
Letters $\mathbb{X}, \mathbb{Y}$ denote Banach spaces, over the field $\mathbb{K} \in\{\mathbb{R}, \mathbb{C}\}$. Let $B_{\mathbb{X}}=\{x \in \mathbb{X}$ : $\|x\| \leq 1\}$ and $S_{\mathbb{X}}=\{x \in \mathbb{X}:\|x\|=1\}$ be the unit ball and the unit sphere of $\mathbb{X}$ respectively. Let $\mathcal{B}(\mathbb{X}, \mathbb{Y})$ and $\mathcal{K}(\mathbb{X}, \mathbb{Y})$ denote the Banach space of all bounded linear operators and compact operators from $\mathbb{X}$ to $\mathbb{Y}$ respectively, endowed with the usual operator norm. We write $\mathcal{B}(\mathbb{X}, \mathbb{Y})=\mathcal{B}(\mathbb{X})$ and $\mathcal{K}(\mathbb{X}, \mathbb{Y})=\mathcal{K}(\mathbb{X})$ if $\mathbb{X}=\mathbb{Y}$. The symbol $I_{\mathbb{X}}$ stands for the identity operator on $\mathbb{X}$. We omit the suffix in case there is no confusion. We reserve the symbol $\mathcal{H}$ for a Hilbert space over the field $\mathbb{K}$. Throughout the paper, we consider only separable Hilbert spaces. In this paper, mostly in the context of bounded linear operators, we discuss three of the most important orthogonality types in a Banach space, namely, Birkhoff-James orthogonality [8] and [13], isosceles orthogonality [12] and Roberts orthogonality [22].

Let us first state the relevant definitions, in the more general setting of a normed space $\mathcal{X}$ over $\mathbb{K}$.

Definition 1.1. For any two elements $x, y \in \mathcal{X}$, we say that $x$ is Birkhoff-James orthogonal to $y$, written as $x \perp_{B} y$, if for all $\lambda \in \mathbb{K}$, the following holds:

$$
\|x\| \leq\|x+\lambda y\|
$$

Definition 1.2. For any two elements $x, y \in \mathcal{X}$ and $\mathbb{K}=\mathbb{R}$, we say that $x$ is isosceles orthogonal to $y$, written as $x \perp_{I} y$, if the following holds:

$$
\|x+y\|=\|x-y\|
$$

In complex normed spaces, we consider the following orthogonality relation

$$
x \perp_{I} y \Leftrightarrow\left\{\begin{aligned}
\|x+y\| & =\|x-y\| \\
\|x+i y\| & =\|x-i y\| .
\end{aligned}\right.
$$

Definition 1.3. For any two elements $x, y \in \mathcal{X}$, we say that $x$ is Roberts orthogonal to $y$, written as $x \perp_{R} y$, if for all $\lambda \in \mathbb{K}$, the following holds:

$$
\|x+\lambda y\|=\|x-\lambda y\| .
$$

It is easy to see that Roberts orthogonality implies Birkhoff-James orthogonality but the converse is not necessarily true.

In order to have a better description of Birkhoff-James orthogonality of bounded linear operators between Banach spaces, we introduce the following notation for any $T, A \in \mathcal{B}(\mathbb{X}, \mathbb{Y}):$

$$
\mathcal{O}_{T, A}=\left\{x \in S_{\mathbb{X}}: T x \perp_{B} A x\right\}
$$


Given $T \in \mathcal{B}(\mathbb{X}, \mathbb{Y})$, define the norm attainment set of $T$ as

$$
M_{T}=\left\{x \in S_{\mathbb{X}}:\|T x\|=\|T\|\right\} .
$$

As observed in [6], 21], 23], 24] and [26], the structure of the norm attainment set of a bounded linear operator is of central importance in studying Birkhoff-James orthogonality and smoothness of the said operator. On the other hand, it was illustrated in [18] that the notion of the norm attainment set of a bounded linear operator is deeply related to the geometry of the space of bounded linear operators between Banach spaces. In the context of $T \in \mathcal{B}(\mathcal{H})$, the corresponding norm attainment set $M_{T}$ was completely characterized in [24]. We would like to remark that Birkhoff-James orthogonality of bounded linear operators on a finite-dimensional Hilbert space $\mathcal{H}$ was completely characterized by Bhatia and $\breve{S}$ emrl in [6]:

$$
\text { For } T, A \in \mathcal{B}(\mathcal{H}), T \perp_{B} A \Longleftrightarrow \mathcal{O}_{T, A} \cap M_{T} \neq \emptyset \text {. }
$$

This motivates us to explore the structure of $\mathcal{O}_{T, A}$, for two given operators $T, A \in$ $\mathcal{B}(\mathbb{X}, \mathbb{Y})$. In order to study the properties of the set $\mathcal{O}_{T, A}$, in the context of a real Banach space, we require the following notions introduced in [23].

Definition 1.4. Let $\mathcal{X}$ be a real normed space. Let $x, y \in \mathcal{X}$. We say that $y \in x^{+}$if $\|x+\lambda y\| \geq\|x\|$ for all $\lambda \geq 0$. Accordingly, we say that $y \in x^{-}$if $\|x+\lambda y\| \geq\|x\|$ for all $\lambda \leq 0$.

In this context we would like to remark that while studying orthogonality of bounded linear operators, Bhattacharyya and Grover [4] also considered the following weaker notion of orthogonality. Let $\mathcal{X}$ be a real or complex normed space and let $x, y \in \mathcal{X}$. We say that $x$ is $r$-orthogonal to $y$, denoted by $x \perp_{B}^{r} y$, if $\|x+\lambda y\| \geq\|x\|$ for all $\lambda \in \mathbb{R}$. Of course, it is trivial to observe that in case $\mathcal{X}$ is real, $x \perp_{B}^{r} y$ if and only if $y \in x^{+}$and $y \in x^{-}$.

The notion of Birkhoff-James orthogonality is intimately connected with the notion of smoothness in Banach spaces. A non-zero element $x \in \mathbb{X}$ is said to be a smooth point if there exists a unique norm one functional $f \in \mathbb{X}^{*}$ such that $f(x)=\|x\|$. We would like to note that the study of smoothness in the space of bounded linear operators between Banach spaces is an active area of interest, and we refer the readers to [19], [21] and [26].

For $A \in \mathcal{B}(\mathcal{H})$, we use the notations $A^{*}, R(A), N(A)$, to denote the adjoint, the range and the kernel of $A$ respectively. If $A, B$ are self-adjoint elements of $\mathcal{B}(\mathcal{H})$, we 
write $A \leq B$ whenever $\langle A x, x\rangle \leq\langle B x, x\rangle$ for all $x \in \mathcal{H}$. An element $A \in \mathcal{B}(\mathcal{H})$ such that $A \geq 0$ is called positive. For every $A \geq 0$, there exists a unique positive $A^{1 / 2} \in \mathcal{B}(\mathcal{H})$ such that $A=\left(A^{1 / 2}\right)^{2}$. For any $\mathcal{B} \subseteq \mathcal{B}(\mathcal{H}), \mathcal{B}^{+}$denotes the subset of all positive operators of $\mathcal{B}$.

For any $T \in \mathcal{B}(\mathcal{H})$, we can write $T=\operatorname{Re}(T)+i \operatorname{Im}(T)$, where $\operatorname{Re}(T)=\frac{T+T^{*}}{2}$ and $\operatorname{Im}(T)=\frac{T-T^{*}}{2 i}$ are self-adjoint operators. This is the so called Cartesian decomposition of $T$.

Let us recall that if $M \subseteq \mathcal{H}$ is a closed subspace of $\mathcal{H}$, then $P_{M}$ denotes the orthogonal projection onto $M$ of $\mathcal{H}$.

For any compact operator $A \in \mathcal{K}(\mathcal{H})$, let $s_{1}(A), s_{2}(A), \cdots$ be the singular values of $A$, i.e., the eigenvalues of the "absolute value-norm" $|A|=\left(A^{*} A\right)^{\frac{1}{2}}$ of $A$, in decreasing order and repeated according to multiplicity.

The notion of unitarily invariant norm (UIN) can be defined for operators on Hilbert spaces as a norm \|\|$. \mid \|$ that satisfies the invariance property $\|U X V\|\|=\|\|X\|$, for any pair of unitary operators $U, V \in \mathcal{B}(\mathcal{H})$. Recall that each UIN is defined on a natural subclass $\mathcal{J} \subseteq \mathcal{K}(\mathcal{H})$, called the norm ideal associated with the norm $\|||$.$\| .$

If $A \in \mathcal{K}(\mathcal{H})$ and $p>0$, let

$$
\|A\|_{p}=\left(\sum_{i=1}^{\infty} s_{i}(A)^{p}\right)^{\frac{1}{p}}=\left(\operatorname{tr}|A|^{p}\right)^{\frac{1}{p}},
$$

where $\operatorname{tr}$ is the usual trace functional, i.e. $\operatorname{tr}(A)=\sum_{j=1}^{\infty}\left\langle A e_{j}, e_{j}\right\rangle$, and $\left\{e_{j}\right\}_{j=1}^{\infty}$ is an orthonormal basis of $\mathcal{H}$. Equality (1.5) defines a norm (quasi-norm) on the ideal $\mathbb{B}_{p}(\mathcal{H})=$ $\left\{A \in \mathcal{K}(\mathcal{H}):\|A\|_{p}<\infty\right\}$ for $1 \leq p<\infty(0<p<1)$, called the $p$-Schatten class.

The study of orthogonality of bounded linear operators is also related to the following notion of operators having disjoint support.

Definition 1.5. Let $\mathcal{H}$ be a real or complex Hilbert space. Two operators $A, B \in \mathcal{B}(\mathcal{H})$ have disjoint support if and only if $A B^{*}=B^{*} A=0$.

We would like to remark that the above definition is not the original one introduced by Arazy in [3], but nevertheless it was proved by Lioudaki in Proposition 2.1.8 of [17] that both notions are equivalent in $\mathbb{B}_{p}(\mathcal{H})$. We also refer the readers to [14] for a related notion of algebraic orthogonality in the setting of $C^{*}$-algebras.

\section{Brief outline of the PAPER}

The main results of this paper are demarcated into three sections. In Section 3, we exclusively study Birkhoff-James orthogonality of bounded linear operators between 
Hilbert spaces and Banach spaces. As mentioned in Remark 3.1 of [6], the finitedimensional Bhatia- $\breve{S}$ emrl theorem can be extended to the setting of infinite-dimensional Hilbert spaces by considering norming sequences for a bounded linear operator, instead of norm attaining vectors corresponding to the said operator. However, we show that even in case of bounded linear operators between infinite-dimensional Banach spaces, it is possible to extend the the finite-dimensional Bhatia- $\breve{S}$ emrl theorem verbatim, under certain additional assumptions. Let us mention here that our observations in this context can be regarded as an extension of Theorem 3.1 and Theorem 4.1 of [28], where only the real case was considered. We also explore the properties of the set $\mathcal{O}_{T, A}=\left\{x \in S_{\mathbb{X}}: T x \perp_{B} A x\right\}$, for any $T, A \in \mathcal{B}(\mathbb{X}, \mathbb{Y})$ and obtain a characterization for a Hilbert space to be finite-dimensional in terms of this newly introduced notion. The study of $\mathcal{O}_{T, A}$ may be regarded as complementary to the study of $M_{T}$ done in [21], [23], [25] and [26]. In Section 4, we focus on orthogonality of bounded linear operators and positive operators on a Hilbert space. We give a complete characterization for isosceles orthogonality of two positive bounded linear operators. In Section 5, we discuss some relations between the two orthogonality types, Birkhoff-James orthogonality and isosceles orthogonality. Our results in this section are valid in the context of any normed space and not just for operators between Banach spaces. We end the present paper by giving examples in the space of bounded linear operators to illustrate that Roberts orthogonality is much stronger (and therefore, restrictive) than either of Birkhoff-James orthogonality and isosceles orthogonality.

\section{Birkhoff-James Orthogonality of Bounded linear operators}

We begin this section by obtaining a verbatim extension of the finite-dimensional Bhatia- $\breve{S}$ emrl theorem to the infinite-dimensional setting, with an additional assumption on the norm attainment set of one of the operators. We would like to remark that such an extension was obtained by Wójcik in [28], in the context of real Banach spaces, with additional geometric assumptions of strict convexity and smoothness on the range space. However, we cover the cases of both real and complex Banach spaces.

Theorem 3.1. Let $\mathbb{X}$ and $\mathbb{Y}$ be Banach spaces, either both real, or, both complex. Let $\mathbb{X}$ be reflexive. Let $T, A \in \mathcal{K}(\mathbb{X}, \mathbb{Y})$ be such that $M_{T}=\left\{ \pm x_{0}\right\}$ in the real case and $M_{T}=\left\{e^{i \theta} x_{0}: \theta \in[0,2 \pi)\right\}$ in the complex case, where $x_{0} \in S_{\mathbb{X}}$. Then $T \perp_{B} A$ if and only if $\mathcal{O}_{T, A} \cap M_{T} \neq \emptyset$.

Proof. The sufficient part of the theorem is trivially true. Let us prove only the necessary part. We will give the proof only for the complex case. The real case can be treated 
similarly, by applying Theorem 2.1 of $[26]$. Since $\mathbb{X}$ is reflexive, $T, A \in \mathcal{K}(\mathbb{X}, \mathbb{Y})$, and $T \perp_{B} A$, it follows from Theorem 2.3 of $[20$ that given any $\alpha \in U=\{\beta \in \mathbb{C}:|\beta|=$ $1, \arg \beta \in[0, \pi)\}$, there exist $x=x(\alpha), y=y(\alpha) \in M_{T}$ such that $A x \in(T x)_{\alpha}^{+}=\{z \in$ $\mathbb{X}:\|T x+\lambda z\| \geq\|T x\| \forall \lambda=t \alpha, t \geq 0\}$ and $A y \in(T y)_{\alpha}^{-}=\{z \in \mathbb{X}:\|T y+\lambda z\| \geq$ $\|T y\| \forall \lambda=t \alpha, t \leq 0\}$. Since $M_{T}=\left\{e^{i \theta} x_{0}: \theta \in[0,2 \pi)\right\}$, we have that $x=e^{i \theta_{1}} x_{0}$ and $y=e^{i \theta_{2}} x_{0}$, for some $\theta_{1}, \theta_{2} \in[0,2 \pi)$. From this, using the linearity of $T$, it is easy to deduce that $T x_{0} \perp_{B} A x_{0}$. In particular, it follows that $\mathcal{O}_{T, A} \cap M_{T} \neq \emptyset$. This completes the proof of the necessary part of the theorem and establishes it completely.

Let us now study the set $\mathcal{O}_{T, A}$, when $T, A \in \mathcal{B}(\mathbb{X}, \mathbb{Y})$ are given. As an immediate application of the set $\mathcal{O}_{T, A}$, in the following proposition, we obtain an easy sufficient condition for Birkhoff-James orthogonality of two bounded linear operators $T, A$ in terms of the set $\mathcal{O}_{T, A}$. The proof of the proposition is omitted as it is rather trivial. We would like to note that the following proposition implies that unless $T \perp_{B} A, \mathcal{O}_{T, A}$ cannot be the whole of $S_{\mathbb{X}}$.

Proposition 3.2. Let $\mathbb{X}, \mathbb{Y}$ be any two Banach spaces, either both real, or, both complex. Let $T, A \in \mathcal{B}(\mathbb{X}, \mathbb{Y})$. If $\mathcal{O}_{T, A}=S_{\mathbb{X}}$ then $T \perp_{B} A$.

On the other hand, in somewhat opposite direction to the above result, we next obtain a necessary condition for Birkhoff-James orthogonality of two compact linear operators $T, A \in \mathcal{K}(\mathbb{X}, \mathbb{Y})$, in terms of the set $\mathcal{O}_{T, A}$, when $\mathbb{X}$ is a reflexive real Banach space and $\mathbb{Y}$ is any real Banach space. We would like to remark that the following theorem is motivated by Theorem 2.1 of [25], with suitable modifications. Therefore, for the sake of brevity, we make use of some of the arguments used in the proof of Theorem 2.1 of [25].

Theorem 3.3. Let $\mathbb{X}$ be a reflexive real Banach space and $\mathbb{Y}$ be any real Banach space. Let $T, A \in \mathcal{K}(\mathbb{X}, \mathbb{Y})$. If $T \perp_{B} A$ then $\mathcal{O}_{T, A} \neq \emptyset$.

Proof. If possible, suppose that $\mathcal{O}_{T, A}=\emptyset$. Therefore, it follows that given any $x \in S_{\mathbb{X}}$, there exists $\lambda_{x} \neq 0$ such that $\left\|T x+\lambda_{x} A x\right\|<\|T x\|$. Let us consider the following two sets:

$$
\begin{aligned}
& V_{1}=\left\{z \in S_{\mathbb{X}}:\|T z+\lambda A z\|<\|T z\| \text { for some } \lambda>0\right\}, \\
& V_{2}=\left\{z \in S_{\mathbb{X}}:\|T z+\lambda A z\|<\|T z\| \text { for some } \lambda<0\right\} .
\end{aligned}
$$

It is easy to check that both $V_{1}$ and $V_{2}$ are open subsets of $S_{\mathbb{X}}$. Applying the convexity of norm, it is also easy to check that $V_{1} \cap V_{2}=\emptyset$. Moreover, it follows from our assumption of $\mathcal{O}_{T, A}=\emptyset$ that $S_{\mathbb{X}}=V_{1} \cup V_{2}$. Since $S_{\mathbb{X}}$ is connected, it follows that either $V_{1}=\emptyset$ or 
$V_{2}=\emptyset$. Now, we will arrive at a contradiction in each of these two cases to complete the proof of the theorem. Since $T, A \in \mathcal{K}(\mathbb{X}, \mathbb{Y})$ and $T \perp_{B} A$, it follows from Theorem 2.1 of [26] that there exists $x, y \in M_{T}$ such that $A x \in(T x)^{+}$and $A y \in(T y)^{-}$. We note that for any $z \in S_{\mathbb{X}}, z \in \mathcal{O}_{T, A}$ if and only if $A z \in(T z)^{+}$and $A z \in(T z)^{-}$. Since we have assumed that $\mathcal{O}_{T, A}=\emptyset$, we must have, $x \in V_{2}$ and $y \in V_{1}$. This proves that $V_{1} \neq \emptyset$ and $V_{2} \neq \emptyset$. This contradiction completes the proof of the theorem.

As another application of the set $\mathcal{O}_{T, A}$, we show that it is possible to characterize whether a given Hilbert space is finite-dimensional, using this concept.

Theorem 3.4. A real or complex Hilbert space $\mathcal{H}$ is finite-dimensional if and only if for any $T, A \in \mathcal{B}(\mathcal{H})$, we have, $T \perp_{B} A \Longrightarrow \mathcal{O}_{T, A} \neq \emptyset$.

Proof. We would like to note that the necessary part of the theorem follows directly from the Bhatia- $\breve{S}$ emrl theorem. Let us prove the sufficient part. If possible, suppose that $\mathcal{H}$ is infinite-dimensional. It follows that there exists a countable orthonormal basis $\left\{e_{n}: n \in \mathbb{N}\right\}$ of $\mathcal{H}$. Define linear operators $T$ and $A$ in $\mathcal{B}(\mathcal{H})$ in the following way: $T e_{1}=\frac{1}{2} e_{1}, T e_{n}=\left(1-\frac{1}{n}\right) e_{n}$ for all $n \geq 2$ and $A e_{n}=\frac{1}{n} e_{n}$. An easy computation reveals that the following are true:

$$
\text { (i) }\|T\|=\|A\|=1, \text { (ii) } T \perp_{B} A \text {, and (iii) } \mathcal{O}_{T, A}=\emptyset .
$$

However, this contradicts our assumption that $T \perp_{B} A \Rightarrow \mathcal{O}_{T, A} \neq \emptyset$. This completes the proof of the theorem.

Remark 3.5. Characterization of inner product spaces among normed spaces is a classical problem in functional analysis. We refer the readers to the excellent book [2] for more information in this regard. In recent times, in connection with the Bhatia$\breve{S}$ emrl theorem, Benítez, Fernández and Soriano [7] have obtained a characterization of finite-dimensional real Hilbert spaces among real Banach spaces. The above theorem is motivated in the same spirit, and it is valid for both real and complex Banach spaces.

From Theorem 2.2 of Sain and Paul [25], it follows that in case of $T \in \mathcal{B}(\mathcal{H})$, where $\mathcal{H}$ is a real or complex Hilbert space, the corresponding norm attainment set $M_{T}$ is either empty or it is the unit sphere of some subspace of $\mathcal{H}$. Motivated by this result, it is natural to pose the following problem:

For which operators $T, A \in \mathcal{B}(\mathcal{H})$, it is true that $\mathcal{O}_{T, A}$ is the unit sphere of some subspace of $\mathcal{H}$ ? 
In the next proposition, we give a sufficient condition for $T, A \in \mathcal{B}(\mathcal{H})$ to be such that $\mathcal{O}_{T, A}$ is the unit sphere of some subspace of $\mathcal{H}$.

Proposition 3.6. Let $\mathcal{H}$ be a real or complex Hilbert space. Let us consider the following set:

$$
\begin{array}{r}
\Gamma=\left\{(T, A) \in \mathcal{B}(\mathcal{H}) \times \mathcal{B}(\mathcal{H}):\left\langle T x_{1}, A x_{2}\right\rangle+\left\langle T x_{2}, A x_{1}\right\rangle=0\right. \\
\text { if } \left.\left\langle T x_{1}, A x_{1}\right\rangle=\left\langle T x_{2}, A x_{2}\right\rangle=0\right\}
\end{array}
$$

Then for any $(T, A) \in \Gamma$, either $\mathcal{O}_{T, A}=\emptyset$ or $\mathcal{O}_{T, A}=S_{M}$, where $M$ is a subspace of $\mathcal{H}$.

Proof. It is enough to prove that if $x_{1}, x_{2}$ satisfy $\left\langle T x_{1}, A x_{1}\right\rangle=\left\langle T x_{2}, A x_{2}\right\rangle=0$ then $\left\langle T\left(x_{1}+x_{2}\right), A\left(x_{1}+x_{2}\right)\right\rangle=0$ and $\left\langle T \lambda x_{1}, A \lambda x_{1}\right\rangle=0$ for all $\lambda \in \mathbb{C}$. We observe that the second condition is trivially true. On the other hand, the first condition holds true since by the hypothesis, we have,

$\left\langle T\left(x_{1}+x_{2}\right), A\left(x_{1}+x_{2}\right)\right\rangle=\left(\left\langle T x_{1}, A x_{1}\right\rangle+\left\langle T x_{2}, A x_{2}\right\rangle\right)+\left(\left\langle T x_{1}, A x_{2}\right\rangle+\left\langle T x_{2}, A x_{1}\right\rangle\right)=0$. This completes the proof of the proposition.

Remark 3.7. It is trivial that if $T, A \in \mathcal{B}(\mathcal{H})$ have disjoint support then $(T, A) \in \Gamma$. However, it is interesting to note that $\Gamma$ contains pairs of operators that do not have disjoint support. Let $M, N$ be finite-dimensional subspaces of $\mathcal{H}$ such that $M \subsetneq N$. We consider $P_{M}$ and $P_{N}$ to be the orthogonal projections on $M$ and $N$ respectively. By the hypothesis, we have that $P_{M} P_{N}=P_{N} P_{M}=P_{M}$. Let us choose $0 \neq x \in M^{\perp} \cap N$ with $\|x\|=1$. It is easy to see that $P_{N} \perp_{B} P_{M}$, since $x \in M_{P_{N}}$ and $\left\langle P_{N} x, P_{M} x\right\rangle=\left\langle P_{M} x, x\right\rangle=0$. On the other hand, $0=\left\langle P_{N} y, P_{M} y\right\rangle=\left\langle P_{M} y, y\right\rangle=\left\|P_{M} y\right\|^{2}$ if and only if $y \in M^{\perp}$. Let $x_{1}, x_{2} \in M^{\perp}$, then $\left\langle P_{N} x_{1}, P_{M} x_{2}\right\rangle+\left\langle P_{N} x_{2}, P_{M} x_{1}\right\rangle=\left\langle P_{M} x_{1}, x_{2}\right\rangle+\left\langle P_{M} x_{2}, x_{1}\right\rangle=0$. Therefore, we have proved that the following three statements hold true:

(i) $\left(P_{N}, P_{M}\right) \in \Gamma($ ii $) P_{N} \perp_{B} P_{M}$ and (iii) $P_{N}, P_{M}$ do not have disjoint support.

\section{Orthogonality in $\mathcal{B}(\mathcal{H})$}

We begin this section by proving that in the context of bounded linear operators on a Hilbert space, disjoint support implies both Birkhoff-James orthogonality and isosceles orthogonality.

Proposition 4.1. Let $A, B \in \mathcal{B}(\mathcal{H})$, where $\mathcal{H}$ is a real or complex Hilbert space, such that $B^{*} A=0$, then the following holds:

(1) $A \perp_{B} B$ and $B \perp_{B} A$. 
(2) $A \perp_{R} B$ and in particular, $A \perp_{I} B$.

Proof. (1) Consider $h \in S_{\mathcal{H}}$. Then for any $\lambda \in \mathbb{K}$

$\|(A+\lambda B) h\|^{2}=\|A h\|^{2}+\|\lambda B h\|^{2}+2|\lambda|^{2} \operatorname{Re}\left\langle B^{*} A h, h\right\rangle=\|A h\|^{2}+\|\lambda B h\|^{2}$,

where $\operatorname{Re}(z)$ denotes the usual real part of $z \in \mathbb{K}$. Therefore, $\|A+\lambda B\|^{2} \geq$ $\|(A+\lambda B) h\|^{2} \geq\|A h\|^{2}$ for all $h \in S_{\mathcal{H}}$, which implies that $\|A+\lambda B\| \geq\|A\|$ for any $\lambda \in \mathbb{K}$. Interchanging the roles of $A$ and $B$, we can obtain in a similar way that $B \perp_{B} A$.

(2) If $A, B \in \mathcal{B}(\mathcal{H})$ satisfy $B^{*} A=0$, then for any $\lambda \in \mathbb{K}$, we have,

$$
\begin{aligned}
\|A+\lambda B\|^{2} & =\sup \left\{\|(A+\lambda B) h\|^{2}: h \in S_{\mathcal{H}}\right\} \\
& =\sup \left\{\|A h\|^{2}+\|\lambda B h\|^{2}: h \in S_{\mathcal{H}}\right\}=\|A-\lambda B\|^{2} .
\end{aligned}
$$

This completes the proof of the second part of the proposition and establishes it completely.

Remark 4.2. (1) In particular, it follows from our previous result that for operators having disjoint support, Birkhoff-James orthogonality relation is symmetric.

(2) From inequality (4.1), we can conclude that if $R(A)$ and $R(B)$ are orthogonal sets then they are Roberts orthogonal operators. We will prove that a similar result holds when we consider any $\|\cdot|\|| \mid$ UIN.

Let $A, B \in \mathcal{J}$, where $\mathcal{J}$ denotes the norm ideal associated with the norm, such that $B^{*} A=0$. It follows that $|B+\lambda A|=|B-\lambda A|$ for all $\lambda \in \mathbb{K}$ and therefore it turns out that $s_{j}(B+\lambda A)=s_{j}(B-\lambda A)$ for any $j \in \mathbb{N}$ and $\|B+\lambda A\| \|=$ $\|B-\lambda A\| \|$.

However, not every pair of operators $A, B \in \mathcal{B}(\mathcal{H})$, such that $A \perp_{B} B$ or $A \perp_{I} B$, have disjoint support. This idea can be illustrated in the next example.

Example 4.3. (1) Finite dimensional case: Let $\mathcal{H}$ be the two-dimensional real Hilbert space. We consider Let $A=\left(\begin{array}{ll}4 & 0 \\ 0 & 3\end{array}\right)$ and $B=\left(\begin{array}{ll}0 & 0 \\ 0 & 1\end{array}\right)$. Then,

(a) $\|A+B\|=\|A-B\|=4$ and

(b) $A^{*} B=\left(\begin{array}{ll}0 & 0 \\ 0 & 3\end{array}\right)$,

which implies that $A, B$ do not have disjoint support.

(2) Infinite dimensional case: Let $\left\{e_{n}\right\}_{n \in \mathbb{N}}$ be an orthonormal basis for $\mathcal{H}$ a complex Hilbert space. Define operators $A, B: \mathcal{H} \rightarrow \mathcal{H}$ such that

$$
A e_{n}=\lambda_{n} e_{n} \text { and } B e_{1}=0, B e_{n}=\lambda_{n} e_{n+1} \forall n \geq 2,
$$


with $\lambda_{n} \in \mathbb{C}$ and $\left|\lambda_{n}\right|=1$. Observe that $\|A x\|=\|x\|$ for all $x \in \mathcal{H}$.

Since $\left\|A e_{1}\right\|=\left\|e_{1}\right\|=1=\|A\|$ and $B e_{1}=0$, it follows that $A \perp_{B} B$. On the other hand, $A$ and $B$ do not have disjoint support, since

$$
A^{*} B e_{n}=A^{*}\left(\lambda_{n} e_{n+1}\right)=\lambda_{n}\left(A^{*} e_{n+1}\right)=\lambda_{n} \overline{\lambda_{n+1}} e_{n+1} \neq 0
$$

Hereafter, unless otherwise mentioned, we consider $\mathcal{H}$ to be a real Hilbert space.

Theorem 4.4. Let $A, B \in \mathcal{B}(\mathcal{H})$ and suppose that there exists $h_{0}, k_{0} \in \mathcal{H}$ such that $h_{0} \in M_{A+B}$ and $k_{0} \in M_{A-B}$. Then the following assertions are true.

(1) If $\left\langle A h_{0}, B h_{0}\right\rangle \leq 0$ and $\left\langle A k_{0}, B k_{0}\right\rangle \geq 0$, then $A \perp_{I} B$.

(2) If $A \perp_{I} B$ then $\left\langle A h_{0}, B h_{0}\right\rangle \geq 0$ and $\left\langle A k_{0}, B k_{0}\right\rangle \leq 0$.

Proof. (1) Assume that all the conditions of the statement are satisfied. Let $f, g$ : $\mathcal{H} \rightarrow \mathbb{R}$ be given by

$$
\begin{aligned}
& f(h)=\|(A+B) h\|^{2}=\|A h\|^{2}+\|B h\|^{2}+2\langle A h, B h\rangle \text { and } \\
& g(h)=\|(A-B) h\|^{2}=\|A h\|^{2}+\|B h\|^{2}-2\langle A h, B h\rangle .
\end{aligned}
$$

Then, $f(h)-g(h)=4\langle A h, B h\rangle$. Suppose that

$$
g\left(k_{0}\right)=\|A-B\|^{2}<\|A+B\|^{2}=f\left(h_{0}\right) \Rightarrow g\left(h_{0}\right) \leq g\left(k_{0}\right)<f\left(h_{0}\right) .
$$

Thus, $0<f\left(h_{0}\right)-g\left(h_{0}\right)=4\left\langle A h_{0}, B h_{0}\right\rangle$, which is a contradiction. Hence, $g\left(k_{0}\right) \geq f\left(h_{0}\right)$. Analogously, it can be proved that $f\left(h_{0}\right) \geq g\left(k_{0}\right)$. Finally,

$$
\|A+B\|^{2}=f\left(h_{0}\right)=g\left(k_{0}\right)=\|A-B\|^{2},
$$

which implies $A \perp_{I} B$.

(2) We only prove the first inequality, the other can be obtained with a similar argument. By the real polarization formula we get

$$
\left\langle A h_{0}, B h_{0}\right\rangle=\frac{1}{4}\left[\left\|(A+B) h_{0}\right\|^{2}-\left\|(A-B) h_{0}\right\|^{2}\right] \geq \frac{1}{4}\left[\|A+B\|^{2}-\|A-B\|^{2}\right]=0 .
$$

Remark 4.5. Suppose that in Theorem 4.4, $h_{0}$ and $k_{0}$ also satisfy

$$
\left\langle A h_{0}, B h_{0}\right\rangle=\left\langle A k_{0}, B k_{0}\right\rangle=0 .
$$

Then, there exists $h_{1} \in S_{\mathcal{H}}$ such that $\left\|(A+B) h_{1}\right\|=\left\|(A-B) h_{1}\right\|$.

It can be easily proved using polarization formula and hypothesis,

$$
0=\left\langle A h_{0}, B h_{0}\right\rangle=\frac{1}{4}\left[\left\|(A+B) h_{0}\right\|^{2}-\left\|(A-B) h_{0}\right\|^{2}\right]
$$


and this implies $\left\|(A-B) h_{0}\right\|=\left\|(A+B) h_{0}\right\|=\left\|(A-B) k_{0}\right\|$, where last equality is due to isosceles orthogonality between $A$ and $B$ previously proved. By a similar argument, it can be proved that $\left\|(A+B) k_{0}\right\|=\left\|(A+B) h_{0}\right\|$. The proof is completed by taking $h_{1} \in\left\{h_{0} ; k_{0}\right\}$.

The following result combines Theorem 4.4 and last remark.

Corollary 4.6. Let $A, B \in \mathcal{B}(\mathcal{H})$ and suppose that there exists $h_{1} \in M_{A+B} \cap M_{A-B}$ such that $\left\langle A h_{1}, B h_{1}\right\rangle=0$. Then $A \perp_{I} B$,

$$
\|A\|^{2}+\|B\|^{2} \leq\|A+B\|^{2}+\|A-B\|^{2} \leq 2\left(\|A\|^{2}+\|B\|^{2}\right),
$$

and if $h_{1} \notin N(A) \cup N(B)$ then $\|A+B\|^{2}=\|A-B\|^{2}=2$.

Proof. It was proved in Theorem 4.4 that, under these hypothesis, $A \perp_{I} B$. Moreover,

$$
\begin{aligned}
& \|A+B\|^{2}=\left\|(A+B) h_{1}\right\|^{2}=\left\|A h_{1}\right\|^{2}+\left\|B h_{1}\right\|^{2} \leq\|A\|^{2}+\|B\|^{2} \text { and } \\
& \|A-B\|^{2}=\left\|(A-B) h_{1}\right\|^{2}=\left\|A h_{1}\right\|^{2}+\left\|B h_{1}\right\|^{2} \leq\|A\|^{2}+\|B\|^{2} .
\end{aligned}
$$

Then,

$$
\|A+B\|^{2}+\|A-B\|^{2} \leq 2\left(\|A\|^{2}+\|B\|^{2}\right)
$$

On the other hand,

$$
\|A\|^{2}+\|B\|^{2} \leq 2 \max \left(\|A\|^{2} ;\|B\|^{2}\right) \leq\|A+B\|^{2}+\|A-B\|^{2},
$$

Finally, it is easy to see that

$$
\left\|(A+B) h_{1}\right\|^{2}\left\|B h_{1}\right\|^{2}-\left\|A h_{1}\right\|^{2}\left\|B h_{1}\right\|^{2}=\left|\left\langle(A+B) h_{1}, B h_{1}\right\rangle\right|^{2}-\left|\left\langle A h_{1}, B h_{1}\right\rangle\right|^{2} .
$$

If we assume that $h_{1} \notin N(A) \cup N(B)$, then $\|A+B\|^{2}=1+\left\|A h_{1}\right\|^{2}$. By symmetry we obtain that $\|A+B\|^{2}=1+\left\|B h_{1}\right\|^{2}$.

Now, by the Parallelogram law we get

$$
\|A+B\|^{2}+\|A-B\|^{2}=\left\|(A+B) h_{1}\right\|^{2}+\left\|(A-B) h_{1}\right\|^{2}=2\left(\left\|A h_{1}\right\|^{2}+\left\|B h_{1}\right\|^{2}\right) .
$$

It follows that $\left\|B h_{1}\right\|=1$ and $\|A+B\|^{2}=2$.

The following result is other characterization of isosceles orthogonality of bounded linear operators in finite-dimensional real Hilbert spaces, with an additional condition.

Theorem 4.7. Let $\mathcal{H}$ be a finite-dimensional real Hilbert space with $\operatorname{dim}(\mathcal{H})=n$ and $A, B \in \mathcal{B}(\mathcal{H})$. Suppose that $M_{A+B}=S_{\mathcal{H}_{1}}, M_{A-B}=S_{\mathcal{H}_{2}}$, and $\operatorname{dim}\left(\mathcal{H}_{1}\right)+\operatorname{dim}\left(\mathcal{H}_{2}\right)>n$. Then, the following statements are equivalent:

(1) $A \perp_{I} B$. 
(2) There exists $x_{0} \in M_{A+B} \cap M_{A-B}$ such that $\left\langle A x_{0}, B x_{0}\right\rangle=0$

Proof. Suppose that statement (1) holds. Since $\operatorname{dim}\left(\mathcal{H}_{1}\right)+\operatorname{dim}\left(\mathcal{H}_{2}\right)>n$, there exists $0 \neq x_{1} \in \mathcal{H}_{1} \cap \mathcal{H}_{2}$. Consider $x_{0}=\frac{x_{1}}{\left\|x_{1}\right\|}$, then $x_{0} \in M_{A+B} \cap M_{A-B}$. Also, by hypothesis and real polarization formula

$$
\left\langle A x_{0}, B x_{0}\right\rangle=\frac{1}{4}\left(\left\|(A+B) x_{0}\right\|^{2}-\left\|(A-B) x_{0}\right\|^{2}\right)=\frac{1}{4}\left(\|A+B\|^{2}-\|A-B\|^{2}\right)=0 .
$$

Conversely, the other implication is a consequence of Corollary 4.6.

Remark 4.8. If $\mathcal{H}$ is a complex Hilbert space, isosceles orthogonality must be defined as in (1.3) and the previous statements can be generalized to this context with proofs which are essentially the same as the real case. For example, in case of a complex Hilbert space $\mathcal{H}$, Theorem 4.7 can be stated in the following way:

Let $A, B \in \mathcal{B}(\mathcal{H})$ with $\operatorname{dim}(\mathcal{H})=n$. Suppose that there exist $\mathcal{W}_{1}, \mathcal{W}_{2}, \mathcal{H}_{1}, \mathcal{H}_{2}$ subspaces of $\mathcal{H}$ such that

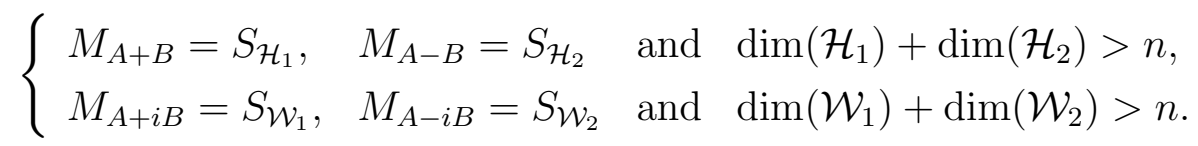

Then, the following statement are equivalent:

(1) $A \perp_{I} B$.

(2) There exist $\left\{\begin{array}{lll}h_{0} \in M_{A+B} \cap M_{A-B} & \text { such that } & \operatorname{Re}\left\langle A h_{0}, B h_{0}\right\rangle=0, \\ k_{0} \in M_{A+i B} \cap M_{A-i B} & \text { such that } & \operatorname{Re}\left\langle A k_{0}, B k_{0}\right\rangle=0 .\end{array}\right.$

In the cone of positive operators between Hilbert spaces, as in a real normed space, we use isosceles orthogonality notion as in (1.2). Kittaneh proved in [16], that if $A, B \in$ $\mathcal{B}(\mathcal{H})^{+}$, then

$$
\begin{aligned}
\max (\|A\|,\|B\|)-\left\|A^{1 / 2} B^{1 / 2}\right\| & \leq\|A-B\| \\
& \leq \max (\|A\|,\|B\|) \leq\|A+B\| \\
& \leq \max (\|A\|,\|B\|)+\left\|A^{1 / 2} B^{1 / 2}\right\| .
\end{aligned}
$$

The above inequalities are useful in the study of isosceles orthogonality in $\mathcal{B}(\mathcal{H})^{+}$. As an immediate consequence, we deduce that if $A, B$ are positve operators and $A^{1 / 2} B^{1 / 2}=0$, then $A \perp_{I} B$.

Proposition 4.9. Let $A, B \in \mathcal{B}(\mathcal{H})^{+}$. Then, the following conditions are equivalent:

(1) $A \perp_{I} B$.

(2) $\|A+B\|=\|A-B\|=\max (\|A\|,\|B\|)$. 
Proof. (1) $\Rightarrow(2)$ Suppose that $A \perp_{I} B$. Equation (4.3) states

$$
\|A-B\| \leq \max (\|A\|,\|B\|) \leq\|A+B\|
$$

for any $A, B \in \mathcal{B}(\mathcal{H})^{+}$. The desired result is now immediate.

The converse implication is trivial.

In order to simplify the exposition, we introduce the following notations. Given $A, B \in \mathcal{B}(\mathcal{H})$ we define

$$
M(A, B)= \begin{cases}A & \text { if } \quad\|B\| \leq\|A\| \\ B & \text { if } \quad\|A\|<\|B\|\end{cases}
$$

and

$$
m(A, B)=\left\{\begin{array}{ll}
B & \text { if } \quad\|B\| \leq\|A\| \\
A & \text { if } \quad\|A\|<\|B\|
\end{array} .\right.
$$

In the next statement we obtain a characterization for isosceles orthogonality when $A$ and $B$ are positive operators.

Theorem 4.10. Let $A, B \in \mathcal{B}(\mathcal{H})^{+}$, then $A \perp_{I} B$ if and only if there exists a sequence $\left\{x_{n}\right\} \subset S_{\mathcal{H}}$ such that $\lim _{n \rightarrow \infty}\left\|M(A, B) x_{n}\right\|=\|A+B\|$ and $\lim _{n \rightarrow \infty} \operatorname{Re}\left\langle B A x_{n}, x_{n}\right\rangle \leq 0$.

Proof. Let $A \perp_{I} B$. By Proposition 4.9 we have $\|A+B\|=\|A-B\|=\max (\|A\|,\|B\|)$. Let $\left\{x_{n}\right\}$ a sequnce of unit vectors in $\mathcal{H}$ such that $\lim _{n \rightarrow \infty}\left\|M(A, B) x_{n}\right\|=\|A+B\|$. Since $\left\{(A+B) x_{n}\right\}$ is a bounded sequence, it has a convergent sequence and without loss of generality we assume that

$$
\lim _{n \rightarrow \infty}\left\|(A+B) x_{n}\right\|^{2} \leq\|A+B\|^{2}=\lim _{n \rightarrow \infty}\left\|M(A, B) x_{n}\right\|^{2} .
$$

Thus $\lim _{n \rightarrow \infty}\left\|m(A, B) x_{n}\right\|^{2}+2 R e\left\langle B A x_{n}, x_{n}\right\rangle \leq 0$ and $\lim _{n \rightarrow \infty} \operatorname{Re}\left\langle B A x_{n}, x_{n}\right\rangle \leq 0$.

Conversely, by the hypothesis there exists a sequence $\left\{x_{n}\right\} \subset S_{\mathcal{H}}$ such that $\|A+B\|=$ $\lim _{n \rightarrow \infty}\left\|M(A, B) x_{n}\right\|$ and $\lim _{n \rightarrow \infty} \operatorname{Re}\left\langle B A x_{n}, x_{n}\right\rangle \leq 0$. From the first condition we have that $\|A+B\|=\max (\|A\|,\|B\|)$. Suppose that $A$ and $B$ are not isosceles orthogonal, this means that $\|A-B\|<\|A+B\|$. Hence, we have

$$
\lim _{n \rightarrow \infty}\left\|(A-B) x_{n}\right\|^{2} \leq\|A-B\|^{2}<\|A+B\|^{2}=\lim _{n \rightarrow \infty}\left\|M(A, B) x_{n}\right\|^{2} .
$$

This implies that $0 \leq \lim _{n \rightarrow \infty}\left\|(A+B) x_{n}\right\|^{2}<\lim _{n \rightarrow \infty} 2 R e\left\langle B A x_{n}, x_{n}\right\rangle$, which is a contradiction. Therefore, $A \perp_{I} B$.

As a consequence of the previous statement, we have the following characterization of the isosceles orthogonal condition for elements of $\mathcal{B}(\mathcal{H})^{+}$such that $\operatorname{Re}(B A) \geq 0$. This extra condition is well known and it is related with acreetive operator theory. We recall 
that an operator $T \in \mathcal{B}(\mathcal{H})$ is called acreetive if in its Cartesian decomposition $\operatorname{Re}(T)$ is positive.

Corollary 4.11. Let $A, B \in \mathcal{B}(\mathcal{H})^{+}$and suppose that $B A$ is acreetive, then $A \perp_{I} B$ if and only if there exists a sequence $\left\{x_{n}\right\} \subset S_{\mathcal{H}}$ such that $\lim _{n \rightarrow \infty}\left\|(A+B) x_{n}\right\|=\|A+B\|$ and $\lim _{n \rightarrow \infty}\left\langle B A x_{n}, x_{n}\right\rangle=0$.

Proof. Let $A \perp_{I} B$. ByTheorem 4.10, there exists a sequence of unit vectors in $\mathcal{H},\left\{x_{n}\right\}$, such that $\lim _{n \rightarrow \infty}\left\|M(A, B) x_{n}\right\|=\|A+B\|$ and $\lim _{n \rightarrow \infty} R e\left\langle B A x_{n}, x_{n}\right\rangle \leq 0$. It follows from

$$
\begin{aligned}
\left\|M(A, B) x_{n}\right\|^{2} & \leq\left\|M(A, B) x_{n}\right\|^{2}+\left\|m(A, B) x_{n}\right\|^{2}+2 \operatorname{Re}\left\langle B A x_{n}, x_{n}\right\rangle \\
& =\left\|(A+B) x_{n}\right\|^{2} \leq\|A+B\|^{2}
\end{aligned}
$$

that $\lim _{n \rightarrow \infty}\left\|(A+B) x_{n}\right\|=\|A+B\|, \lim _{n \rightarrow \infty}\left\|m(A, B) x_{n}\right\|=0$ and $\lim _{n \rightarrow \infty} \operatorname{Re}\left\langle B A x_{n}, x_{n}\right\rangle=0$.

Since $\left|\left\langle B A x_{n}, x_{n}\right\rangle\right| \leq\left\|M(A, B) x_{n}\right\|\left\|m(A, B) x_{n}\right\|$ we infer that $\lim _{n \rightarrow \infty}\left\langle B A x_{n}, x_{n}\right\rangle=0$.

Conversely, by the hypothesis there exists a sequence $\left\{x_{n}\right\} \subset S_{\mathcal{H}}$ such that $\lim _{n \rightarrow \infty} \|(A+$ $B) x_{n}\|=\| A+B \|$ and $\lim _{n \rightarrow \infty}\left\langle B A x_{n}, x_{n}\right\rangle=0$. Suppose that $A$ and $B$ are not isosceles orthogonal, then $\|A-B\|<\|A+B\|$. Hence we have

$$
\lim _{n \rightarrow \infty}\left\|(A-B) x_{n}\right\|^{2} \leq\|A-B\|^{2}<\|A+B\|^{2}=\lim _{n \rightarrow \infty}\left\|(A+B) x_{n}\right\|^{2} .
$$

This implies that $0<4 \lim _{n \rightarrow \infty} \operatorname{Re}\left\langle B A x_{n}, x_{n}\right\rangle$, which is contradiction. Therefore, $A \perp_{I} B$.

Corollary 4.12. Let $A, B \in \mathcal{B}(\mathcal{H})^{+}$and suppose that $B A$ is acreetive. If $A \perp_{I} B$ then $A \perp_{B} B$ or $B \perp_{B} A$.

Remark 4.13. Let $A, B \in \mathcal{B}(\mathcal{H})$ such that $A, B>0$ (i.e. positive and invertible). We will prove that $A$ can not be isosceles orthogonal to $B$. Indeed, suppose that $A \perp_{I} B$. Using formulas (26) and (27) in [16] we have

$$
\begin{gathered}
\max (\|A\|,\|B\|)+\min \left(\left\|A^{-1}\right\|^{-1},\left\|B^{-1}\right\|^{-1}\right) \leq\|A+B\| \\
=\|A-B\| \leq \max (\|A\|,\|B\|)-\min \left(\left\|A^{-1}\right\|^{-1},\left\|B^{-1}\right\|^{-1}\right) \\
\Rightarrow 0<2 \min \left(\left\|A^{-1}\right\|^{-1},\left\|B^{-1}\right\|^{-1}\right) \leq 0
\end{gathered}
$$

which is a contradiction.

A natural question is whether $A$ and $B$ can be Birkhoff-James orthogonal. Suppose that $A \perp_{B} B$, then for any $\lambda>0$, we have,

$$
\|A\| \leq\|A-\lambda B\| \leq \max (\|A\|, \lambda\|B\|)-\min \left(\left\|A^{-1}\right\|^{-1}, \lambda\left\|B^{-1}\right\|^{-1}\right)<\max (\|A\|, \lambda\|B\|),
$$


which is clearly a contradiction. This proves that $A$ is not Birkhoff-James orthogonal to $B$.

Next results and comments are related to isosceles orthogonality between positive operators and projections.

Proposition 4.14. For any $P \in \mathcal{B}(\mathcal{H})^{+}, P \perp_{I} I$ if and only if $P=0$.

Proof. By Proposition 4.9, $\|P-I\|=\|P+I\|=\max \{1 ;\|P\|\}$. Suppose $\|P\| \geq 1$. Then $\|P-I\|=\|P+I\|=\|P\|$, which is a contradiction since $\|(P+I) x\|^{2}>\|P x\|^{2}$ for all $x \neq 0$. On the other hand, if $\|P\|<1$ and $P \neq 0$, consider $x \neq 0,\|x\|=1$ and $P x \neq 0$. Then,

$$
\begin{gathered}
0 \leq\|P x+x\|^{2}=\|P x\|^{2}+2\langle P x, x\rangle+\|x\|^{2} \leq 1 \Rightarrow\|P x\|^{2}+2\langle P x, x\rangle=0 \\
\Rightarrow\|P x\|^{2}=2\langle P x, x\rangle=0 .
\end{gathered}
$$

This completes the proof.

From the above, in finite-dimensional context, we deduce if $A=U P \neq 0$, with $U$ unitary and $P \geq 0$, then $A$ and $U$ can not be isosceles orthogonal. Moreover, using Theorem IX.7.2 in [5], we obtain that $\|A-U\|<\|A+U\|$.

In case of orthogonal projections isosceles orthogonality implies disjoint support, as we show in the next result.

Proposition 4.15. Let $P_{S}, P_{T}$ be orthogonal projections with $S \neq T$. Then, $P_{S} \perp_{I} P_{T}$ if and only if $P_{S} P_{T}=0$. In particular, if $P_{S} \perp_{I} P_{T}$ then $P_{S} \perp_{B} P_{T}$ and $P_{T} \perp_{B} P_{S}$.

Proof. Suppose $P_{S} \perp_{I} P_{T}$. By equation (4.3),

$$
\left\|P_{T}-P_{S}\right\| \leq \max \left(\left\|P_{S}\right\|,\left\|P_{T}\right\|\right) \leq\left\|P_{S}+P_{T}\right\|
$$

Then by hypothesis, $\left\|P_{S}-P_{T}\right\|=\left\|P_{S}+P_{T}\right\|=1$. On the other hand, by [15], $\| P_{S}-$ $P_{T} \|=1$ if and only if $P_{T}$ and $P_{S}$ commute and, by [11], we have $P_{T} P_{S}=P_{S} P_{T}=P_{T \cap S}$. If there exists $h \in T \cap S$ with $h \neq 0$, then $\left\|\left(P_{T}+P_{S}\right) h\right\|=2\|h\|$ and this implies $\left\|P_{T}+P_{S}\right\| \geq 2$, which is a contradiction. Therefore, $T \cap S=\{0\}$ and $P_{S} P_{T}=P_{\{0\}}=0$.

However, it is not true that there exists a equivalence between disjoint support and Birkhoff-James orthogonality, even in the case of orthogonal projections. For instance, consider in $\mathbb{R}^{3}$ the projections onto the planes $z=0$ and $x=0, P_{z=0}$ and $P_{x=0}$, respectively. Clearly, $P_{z=0} \perp_{B} P_{x=0}$ but $P_{z=0} \cap P_{x=0}=P_{x=0, z=0} \neq 0$, which means they have not disjoint support. 


\section{Relations BetweEn DifFERENT TYPES OF ORTHOGONALity}

In this short section we study the relations between Birkhoff-James orthogonality and isosceles orthogonality. Before proceeding any further, let us mention the following fact that serves as a motivation behind our exploration in this section.

Bottazzi et. al. studied the equivalence of Birkhoff-James orthogonality and isosceles orthogonality of positive operators $A, B$ in a $p$-Schatten ideal in [9]. Indeed, for every $A, B \in \mathcal{B}(\mathcal{H})^{+}$and $1<p \leq 2$, they proved that $A \perp_{B}^{p} B$ and $A \perp_{I}^{p} B$ are equivalent notions.

However, it is not difficult to observe that there are many examples in $\mathcal{B}(\mathcal{H}$ ) (and more generally, in Banach spaces) that show $\perp_{B}$ and $\perp_{I}$ are independent orthogonality types and none of them imply the other. Our purpose in this section is to establish relations between these two orthogonality types, in the sense that we determine which additional conditions may be required to have " $\perp_{B} \Rightarrow \perp_{I}$ " and vice versa. Recall that $\mathcal{X}$ is a real or complex normed space.

Proposition 5.1. Let $x, y \in \mathcal{X}$ and assume that $(x+y) \perp_{B} y$ and $(x-y) \perp_{B} y$. Then $x \perp_{I} y$.

Proof. By the hypothesis, we have, $\|x+y\| \leq\|x+y+\lambda y\| \forall \lambda \in \mathbb{K}$. Taking $\beta=1+\lambda$, we have, $\|x+y\| \leq\|x+\beta y\|$. In particular for $\beta=-1$, we get $\|x+y\| \leq\|x-y\|$. Analogously, from the hypothesis $(x-y) \perp_{B} y$, we obtain $\|x-y\| \leq\|x+y\|$. This proves that $x \perp_{I} y$ and completes the proof of the proposition.

In order to address the converse question, we introduce the concept of strongly isosceles orthogonality in real Banach spaces.

Definition 5.2. Let $x, y \in \mathcal{X}$. We say that $x$ is strongly isosceles orthogonal to $y$, written as $x \perp_{S I} y$ if

(1) $x \perp_{I} y$.

(2) there exists a real sequence $\left\{\lambda_{n}\right\}_{n \in \mathbb{N}}$, with $\lambda_{n}>0$, such that $\lim _{n \rightarrow \infty} \lambda_{n}=0$ and $x \perp_{I} \lambda_{n} y$ for all $n \in \mathbb{N}$.

In view of the above definition, we obtain the following statement.

Theorem 5.3. Let $x, y \in \mathcal{X}$. Then $x \perp_{S I} y$ implies $x \perp_{B}^{r} y$ and in particular if $\mathcal{X}$ is a real normed space then $x \perp_{S I} y$ implies $x \perp_{B} y$.

Proof. By Theorem 4.1 of [12], it follows that $\|x\| \leq\|x+\lambda y\|$ for $\lambda \in \mathbb{R},|\lambda| \geq 1$. Therefore, we only have to prove the Birkhoff-James orthogonality condition for $|\lambda|<1$. 
Let $\lambda_{0} \in \mathbb{R}$ such that $\left|\lambda_{0}\right|<1$. By the hypothesis, there exists $n_{0} \in \mathbb{N}$ such that $0<\lambda_{n_{0}}<\left|\lambda_{0}\right|$ and $x \perp_{I} \lambda_{n_{0}} y$. Then, $\beta=\frac{\lambda_{0}}{\lambda_{n_{0}}}$ satisfies $|\beta|>1$ and, by the cited result of James [12], we get $\|x\| \leq\left\|x+\beta \lambda_{n_{0}} y\right\|$, since $|\beta|>1$ and $x \perp_{I} \lambda_{n_{0}} y$. However, this is clearly equivalent to the following:

$$
\|x\| \leq\left\|x+\frac{\lambda_{0}}{\lambda_{n_{0}}} \lambda_{n_{0}} y\right\|=\left\|x+\lambda_{0} y\right\| .
$$

This completes the proof of the proposition.

Remark 5.4. Using the convexity of the norm function, it is possible to show that only condition (ii) in the definition of strongly isosceles orthogonality is sufficient to ensure Birkhoff-James orthogonality of the corresponding elements. However, we include condition (i) in the definition of strongly isosceles orthogonality because we are trying to address the question that asks Isosceles orthogonality, along with which additional conditions, implies Birkhoff-James orthogonality.

We have already discussed that Roberts orthogonality is stronger and more restrictive than either of Birkhoff-James orthogonality and isosceles orthogonality. Moreover, it is obvious that $A \perp_{R} B \Rightarrow A \perp_{S I} B$. In the next two examples we show that the converse of this statement is not necessarily true. We deliberately give the examples using different norms on $\mathcal{B}(\mathcal{H})$, to make them more illustrative.

Example 5.5. Let $\mathcal{H}$ be the two-dimensional real Hilbert space. Consider the Banach space $\mathcal{B}(\mathcal{H})$, endowed with the usual uniform norm. Let $A$ and $B$ matrices considered in Example 4.3 item (1). Then,

(1) $\|A+B\|=\|A-B\|=4$

(2) Let $\left\{\lambda_{n}\right\}_{n \in \mathbb{N}}$ be a sequence such that $\lambda_{n} \in(0,1)$ and $\lambda_{n} \rightarrow 0$. We have,

$$
A+\lambda_{n} B=\left(\begin{array}{cc}
4 & 0 \\
0 & 3+\lambda_{n}
\end{array}\right) \Rightarrow\left\|A+\lambda_{n} B\right\|=4
$$

On the other hand,

$$
A-\lambda_{n} B=\left(\begin{array}{cc}
4 & 0 \\
0 & 3-\lambda_{n}
\end{array}\right) \Rightarrow\left\|A-\lambda_{n} B\right\|=4 .
$$

Conditions (1) and (2) together imply that $A \perp_{S I} B$.

(3) However, if we consider $\lambda=5$, we have

$$
A+5 B=\left(\begin{array}{ll}
4 & 0 \\
0 & 8
\end{array}\right) \Rightarrow\|A+5 B\|=8 \text { and } A-5 B=\left(\begin{array}{cc}
4 & 0 \\
0 & -2
\end{array}\right) \Rightarrow\|A-5 B\|=4 \text {. }
$$

Therefore, $A$ is not Roberts orthogonal to $B$. 
Example 5.6. Let $\mathcal{H}$ be the two-dimensional real Hilbert space. Consider the Banach space $\mathcal{B}(\mathcal{H})$, but now endowed with the 1 -Schatten norm.

$$
\begin{aligned}
& \text { Let } A=\left(\begin{array}{cc}
1 & 0 \\
0 & -2
\end{array}\right) \text { and } I=\left(\begin{array}{ll}
1 & 0 \\
0 & 1
\end{array}\right) \in \mathcal{B}(\mathcal{H}) \text {. Then, } \\
& \text { (1) }\|A+I\|_{1}=2+1=\|A-I\|_{1} \text {. } \\
& \text { (2) Let }\left\{\lambda_{n}\right\}_{n \in \mathbb{N}} \text { be a sequence such that } \lambda_{n} \in(0,1) \text { and } \lambda_{n} \rightarrow 0, \\
& A+\lambda_{n} I=\left(\begin{array}{cc}
1+\lambda_{n} & 0 \\
0 & -2+\lambda_{n}
\end{array}\right) \Rightarrow\left\|A+\lambda_{n} B\right\|_{1}=\left|1+\lambda_{n}\right|+\left|-2+\lambda_{n}\right|=3 \\
& A-\lambda_{n} I=\left(\begin{array}{cc}
1-\lambda_{n} & 0 \\
0 & -2-\lambda_{n}
\end{array}\right) \Rightarrow\left\|A-\lambda_{n} B\right\|_{1}=\left|1-\lambda_{n}\right|+\left|-2-\lambda_{n}\right|=3 .
\end{aligned}
$$

Clearly, conditions (1) and (2) together imply that $A \perp_{S I} I$.

(3) But if we consider $\lambda=2$, we have,

$$
A+2 I=\left(\begin{array}{ll}
3 & 0 \\
0 & 0
\end{array}\right) \Rightarrow\|A+2 I\|_{1}=3 \text { and } A-2 I=\left(\begin{array}{cc}
-1 & 0 \\
0 & -4
\end{array}\right) \Rightarrow\|A-2 I\|_{1}=5 .
$$

This proves that $A$ is not Roberts orthogonal to $B$.

\section{Acknowledgements}

The research of Dr. Tamara Bottazzi and Dr. Cristian Conde is partially supported by National Scientific and Technical Resesarch Conuncil of Argentina (CONICET)and ANPCyT PICT 2017-2522.

The research of Dr. Debmalya Sain is sponsored by Dr. D. S. Kothari Post-doctoral Fellowship, under the mentorship of Professor Gadadhar Misra. Dr. Sain feels elated to acknowledge the wonderful hospitality of the lovely couple Mr. Subhro Jana and Mrs. Poulomi Mallik.

\section{REFERENCES}

[1] Alonso J., Martini H., Wu S., On Birkhoff orthogonality and isosceles orthogonality in normed linear spaces, Aequationes Math., 83, 153-189 (2012).

[2] Amir D., Characterizations of Inner Product Spaces, Operator Theory: Advances and Applications, 20, Birkhäuser, Basel (1986).

[3] Arazy J., The isometries of $C_{p}$, Israel J. Math., 22 no. 3-4, 247-256 (1975).

[4] Bhattacharyya T., Grover P., Characterization of Birkhoff-James orthogonality, J. Math. Anal. Appl., 407 no. 2, 350-358 (2013).

[5] Bhatia R., Matrix analysis, Graduate Texts in Mathematics, 169, Springer-Verlag, New York (1997). 
[6] Bhatia R., Šemrl P., Orthogonality of matrices and some distance problems, Linear Algebra Appl., 287, 77-85 (1999).

[7] Benítez C., Fernández M., Soriano M. L., Orthogonality of matrices, Linear Algebra Appl., 422, 155-163 (2007).

[8] Birkhoff G., Orthogonality in linear metric spaces, Duke Math. J., 1 no. 2, 169-172 (1935).

[9] Bottazzi T., Conde C., Moslehian M. S, Wójcik P., Zamani A., Orthogonality and parallelism of operators on various Banach spaces, J. Aust. Math. Soc., 106 no. 2, 160-183 (2019).

[10] Chmieliński J., Operators reversing orthogonality in normed spaces, Adv. Oper. Theory, 1 no. 1, 8-14 (2016).

[11] Deutsch F., The angle between subspaces of a Hilbert space, NATO Adv. Sci. Inst. Ser. C Math. Phys. Sci., 454, 107-130 (1995).

[12] James R. C., Orthogonality in normed linear spaces, Duke Math. J., 12, 291-302 (1945).

[13] James R. C., Orthogonality and linear functionals in normed linear spaces, Trans. Amer. Math. Soc., 61, 265-292 (1947).

[14] Karn A. K., Orthogonality in $C^{*}$ - algebras, Positivity, 20 no. 3, 607-620 (2016).

[15] Kato T., Perturbation theory for linear operators, Classics in Mathematics, Springer-Verlag, Berlin (1995).

[16] Kittaneh F., Norm inequalities for sums and differences of positive operators, Linear Algebra Appl., 383, 85-91 (2004).

[17] Lioudaki V., Operator theory on $C_{p}$ spaces (doctoral dissertation), University of Edinburgh (2004). https://www.era.lib.ed.ac.uk/handle/1842/12432

[18] Mal A., Sain D., Paul K., On some geometric properties of operator spaces, Banach J. Math. Anal., 13 no. 1, 174-191 (2019).

[19] Paul K., Sain D., Ghosh P., Birkhoff-James orthogonality and smoothness of bounded linear operators, Linear Algebra Appl., 506, 551-563 (2016).

[20] Paul K., Sain D., Mal A., Mandal K., Orthogonality of bounded linear operators on complex Banach spaces, Adv. Oper. Theory, 3 no. 3, 699-709 (2018).

[21] Rao T. S. S. R. K., Smooth points in spaces of operators, Linear Algebra Appl., 517, 129-133 (2017).

[22] Roberts B., On the geometry of abstract vector spaces, Tohoku Math. J., 39, 42-59 (1934).

[23] Sain D., Birkhoff-James orthogonality of linear operators on finite dimensional Banach spaces, J. Math. Anal. Appl., 447 no. 2, 860-866 (2016).

[24] Sain D., On extreme contractions and the norm attainment set of a bounded linear operator, Ann. Funct. Anal., 10 no. 1, 135-143 (2019).

[25] Sain D., Paul K., Operator norm attainment and inner product spaces, Linear Algebra Appl., 439 no. 8, 2448-2452 (2013).

[26] Sain D., Paul K., Mal A., A complete characterization of Birkhoff-James orthogonality in infinite dimensional normed space, J. Operator Theory, 80 no. 2, 399-413 (2018).

[27] Turnšek A., A remark on orthogonality and symmetry of operators in $\mathcal{B}(\mathcal{H})$, Linear Algebra Appl., $535,141-150$ (2017).

[28] Wójcik P., Orthogonality of compact operators, Expositiones Mathematicae, 35, 86 - 94 (2017). 
[29] Zamani A., Birkhoff-James orthogonality of operators in semi-Hilbertian spaces and its applications, Ann. Funct. Anal., 10 no. 3, 433-445 (2019).

1 Sede Andina, Universidad Nacional de Río Negro, (8400) S.C. de Bariloche, ArGENTINA

${ }^{2}$ Instituto Argentino de Matemática "Alberto P. Calderón”, Saavedra 15 3er. Piso, (C1083ACA) Buenos Aires, Argentina

${ }^{3}$ Instituto de Ciencias, Universidad Nacional de Gral. Sarmiento, J. M. Gutierrez 1150, (B1613GSX) Los Polvorines, Argentina

${ }^{4}$ Indian Institute of Science, Bengaluru, Karnataka, India

E-mail address: tbottazzi@unrn.edu.ar

E-mail address: cconde@ungs.edu.ar

E-mail address: saindebmalya@gmail.com 